\title{
Visible spectroscopy of the new ESO large program on trans-Neptunian objects and Centaurs ${ }^{\star}$
}

\author{
Part 1 \\ A. Alvarez-Candal ${ }^{1}$, S. Fornasier ${ }^{1,2}$, M. A. Barucci ${ }^{1}$, C. de Bergh ${ }^{1}$, and F. Merlin ${ }^{1}$ \\ 1 LESIA, Observatoire de Paris, 92195 Meudon Pricipal Cedex, France \\ e-mail: alvaro.alvarez@obspm.fr \\ ${ }^{2}$ Université de Paris 7 Denis Diderot, France
}

Received 3 March 2008 / Accepted 20 May 2008

\section{ABSTRACT}

\begin{abstract}
Aims. A second large observational program was started to improve our knowledge of the physical properties of Centaurs and transNeptunian objects, TNOs. The program is being executed in the facilities at the Cerro Paranal of the European Southern Observatory, ESO, in Chile.

Methods. Visible spectroscopy was carried out using FORS1 at the UT2 of the VLT telescope. We computed the spectral slope, $S$, for each object, and we searched for the possible existence of weak absorption features. We compared our data with those from the literature.

Results. We present spectra for 21 objects, 7 of them with no previously reported spectra. Our sample includes 5 Centaurs, 5 resonant objects, 8 classical TNOs, 2 detached objects, and 1 scattered disk object.

There are significant differences between the distribution of spectral slopes for Centaurs and classical TNOs. The data presented here generally agree with previous published data. One exception is 60558 Echeclus, whose spectral slope is considerably smaller than previous measurements. Another interesting object is $47932\left(2000 \mathrm{GN}_{171}\right)$, which does not show evidence of a proposed feature at $0.7 \mu \mathrm{m}$.

Most of the spectra are linear and featureless; however, the well-known bands of solid methane are detected in 136199 Eris spectra and some weak features are detected for a few other objects. The most interesting case is that of $2003 \mathrm{AZ}_{84}$, which has a feature at about $0.65 \mu \mathrm{m}$, maybe due to aqueous alteration.
\end{abstract}

Key words. techniques: spectroscopic - Kuiper Belt

\section{Introduction}

More than a thousand objects have been discovered to date in the trans-Neptunian region. Different techniques covering different wavelength ranges have been used to study them.

One of the most used techniques is visible spectroscopy, ranging approximately from 0.4 up to $0.9 \mu \mathrm{m}$. In general, the observed spectra show a linear trend without evident features, corresponding to colors ranging from bluish-neutral, i.e., solar colors, to red. Few exceptions are known. The largest objects, such as 134340 Pluto, 136199 Eris, and 136472 (2005 FY9), show absorption features in the visible due to methane ice. Tegler et al. (2007) detected a hint of a band at $0.5773 \mu \mathrm{m}$, possibly due to $\mathrm{O}_{2}$ ice on the spectrum of $2003 \mathrm{EL}_{61}$, but due to the low signal-to-noise of the spectrum, confirmation is required with higher continuum signal precision. For a detailed discussion on the largest TNOs, see Brown (2008).

Why observe objects that apparently do not show any evidence of absorption features, unless they have diameters greater than $2000 \mathrm{~km}$ ? One main objective is to analyze the spectral

\footnotetext{
* Based on observations at the VLT Observatory Cerro Paranal of European Southern Observatory, ESO, Chile, in the framework of program 178.C-0036.
}

slopes that are indicative of the composition of the surfaces. Another is to try to find possible relations between them and the orbital parameters of the different objects. It is also possible to find subtle features, such as those due to hydrated minerals, similar to those found in asteroids from the Main Belt (Vilas et al. 1993). One such example is the features found on $2003 \mathrm{AZ}_{84}$ (Fornasier et al. 2004a) or $47932\left(2000 \mathrm{GN}_{171}\right)$ (Lazzarin et al. 2003; de Bergh et al. 2004).

To investigate the surface properties of these primitive objects, a new large program was started at ESO (in Cerro Paranal and La Silla) in October 2006 (PI M.A. Barucci). The program involves the utilization of different instruments in an attempt to obtain quasi-simultaneous photometry and spectroscopy, as much as is possible, covering the range from 0.4 up to $2.5 \mu \mathrm{m}$. Here we present the first results of the visible spectroscopy obtained with the FORS1 instrument, placed at the UT2 (KUEYEN) unit of the VLT telescope. We observed 21 objects of different dynamical classes (see Table 1). Seven of these objects have their spectra presented for the first time: 42355 Typhon, 55637 (2002 $\left.\mathrm{UX}_{25}\right), 119951$ (2002 $\left.\mathrm{KX}_{14}\right)$, $120132\left(2003 \mathrm{FY}_{128}\right), 145452\left(2005 \mathrm{RN}_{43}\right), 2003 \mathrm{QW}_{90}$, and $2003 \mathrm{UZ}_{117}$. The observations are described in the next section. In Sect. 3 we present the main results, which are discussed in 
Sect. 4. Finally, in Sect. 5 we present the conclusions with a short summary of the results.

\section{Observations and data reduction}

The data were obtained during two runs in visitor mode on 22-24 January 2007 and 18, 19 September 2007, and 5 servicemode runs from October 2006 to July 2007. Details on the spectroscopic observations are given in Table 1. For each object we report the dynamical class, heliocentric and geocentric distance, phase angle, observing mode, observational date and universal time (UT of the beginning of the exposure), the total exposure time, the average airmass and seeing, and the observed solar analog stars used to remove the telluric and solar contributions. The last columns show the spectral slope and the departure from linearity of the spectra (see below for the definition).

All observations were obtained with the FORS1 instrument using a low resolution grism (150 grooves $/ \mathrm{mm}$ ) with a $1^{\prime \prime}$ wide slit, covering the $0.4-0.94 \mu \mathrm{m}$ wavelength range ${ }^{1}$ with a spectral resolution of about 200 . The slit was oriented along the parallactic angle to minimize the effects of atmospheric differential refraction, which is also instrumentally corrected by a system of two silica prisms (named Longitudinal ADC).

For the October 2006-February 2007 observations, the FORS1 detector was a TK2048EB4-1 backside-thinned CCD of $2048 \times 2048$ pixel, with a pixel size of $24 \mu \mathrm{m}$, corresponding to a pixel scale of $0.2 \mathrm{arcsec} / \mathrm{px}$; the high gain mode (1.45 $\left.\mathrm{e}^{-} / \mathrm{ADU}\right)$ was used for the read out of the CCD. In April 2007 the FORS1 instrument was upgraded and the detector was replaced with a new mosaic of two $2 \mathrm{k} \times 4 \mathrm{k}$ E2V CCDs (with $15 \mu \mathrm{m}$ pixels) optimized for the blue range $(<0.6 \mu \mathrm{m})$, and with a strong fringing for wavelengths $>0.65 \mu \mathrm{m}$. We always use the 150 grooves $/ \mathrm{mm}$ grism. The image scale is about $0.25 \mathrm{arcsec} / \mathrm{px}$ for the spectroscopic mode. The sorting filter $O G 435$ was used to eliminate the second order contribution during visitor mode and March 2007 service mode observations. The observations done in service mode during October-December 2006 and during May and July 2007 were made without a filter. During each night we also acquired bias, flat-fields, calibration lamps (He-Ar), and several solar analog stars spectra at different intervals throughout the night.

The spectra were reduced using standard data reduction procedures (see Fornasier et al. 2004b) with the software packages Midas and IRAF. To minimize the effect of fringing in all the spectra taken after April 2007, we split a single exposure into two or three exposures taken with an arbitrary offset and then combined them to obtain a single spectrum. Wavelength calibration was performed using helium and argon lamp spectral lines. The precision of the calibration is about $0.0004 \mu \mathrm{m}$. The reflectivity of the objects was obtained by dividing their spectra by the spectrum of the solar-analog star closest in time and airmass, as reported in Table 1.

Spectra were normalized to one at $0.55 \mu \mathrm{m}$ and finally smoothed with a median filter technique, using a box of $0.0039 \mu \mathrm{m}$ width in the spectral direction for each point of the spectrum. The threshold was set to 0.1 , meaning that the original value was replaced by the median value when this last differed by more than $10 \%$ from the original one. All the obtained spectra are shown in Figs. 1-5. Note that in the figures photometric data also appear taken just before or after the corresponding spectrum. These photometric data will be discussed in a forthcoming

\footnotetext{
${ }^{1}$ Useful range for the study presented here.
}

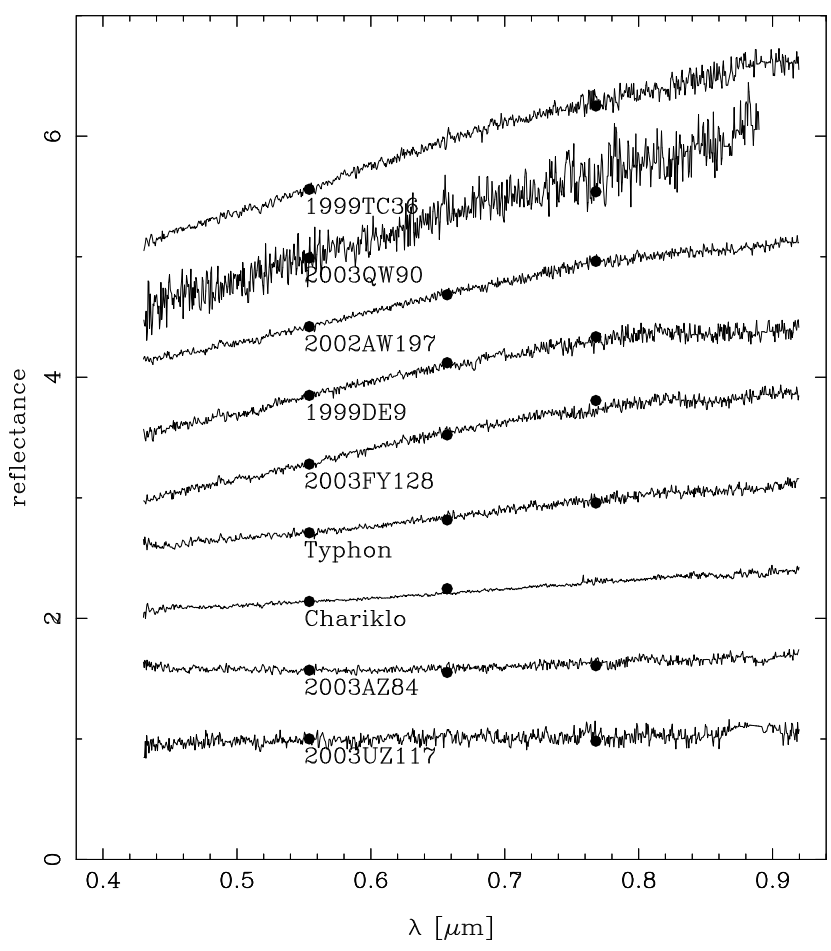

Fig. 1. Visible spectra of 9 objects taken before April 2007 with the old detector of FORS1. Spectra are shifted vertically by 0.57 for clarity.

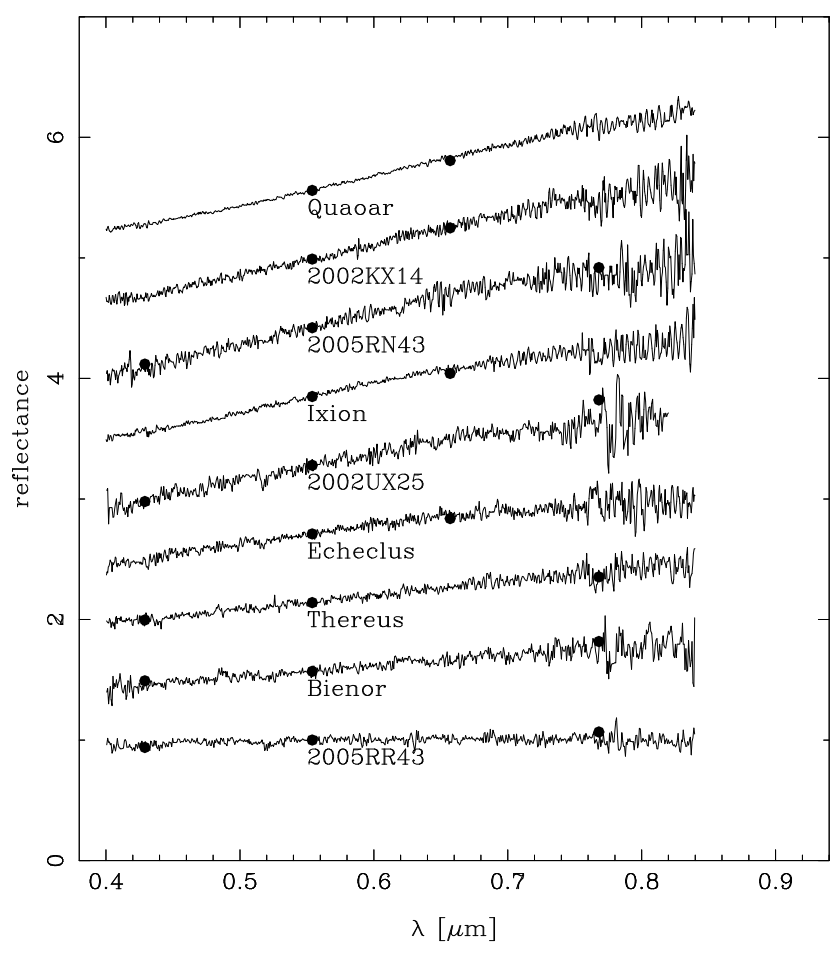

Fig. 2. Visible spectra of 9 objects taken after April 2007 with the new detector of FORS1. Spectra are shifted vertically by 0.57 for clarity.

paper (DeMeo et al. 2008) and are shown here in order to see the agreement between the spectra and the colors.

\subsection{New vs. old detector}

As mentioned above, in April 2007 FORS1 was upgraded, receiving a new detector that was more efficient in the blue. In the 


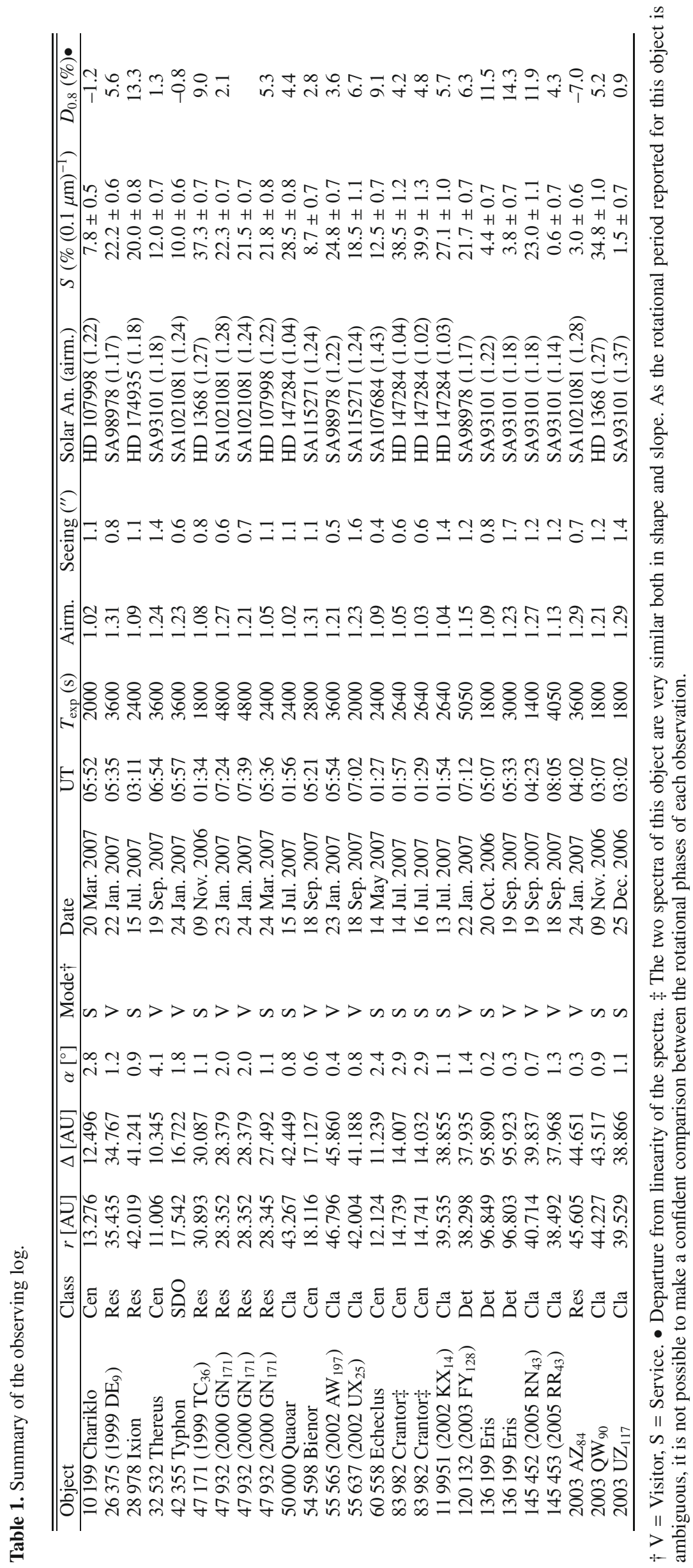




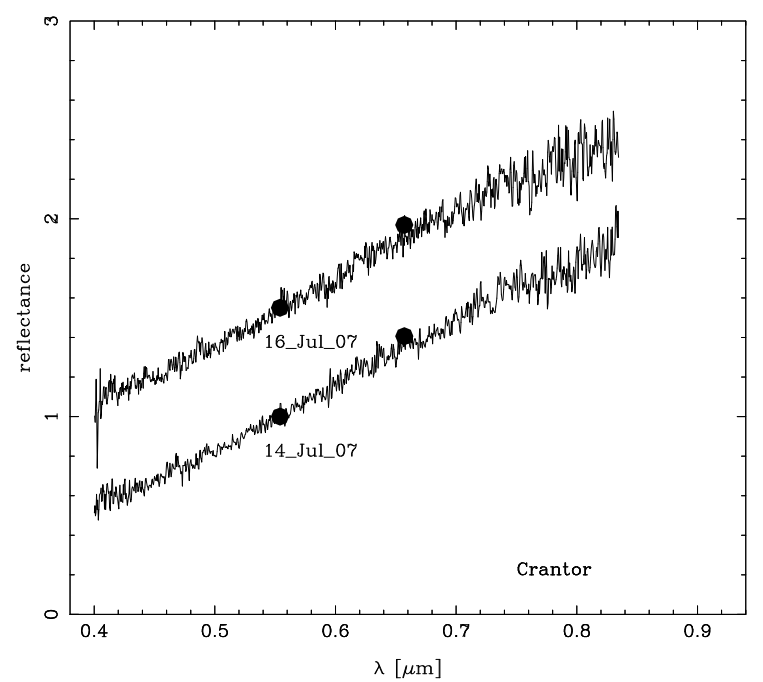

Fig. 3. Visible spectra of 83982 Crantor. Spectra are shifted vertically by 0.55 .

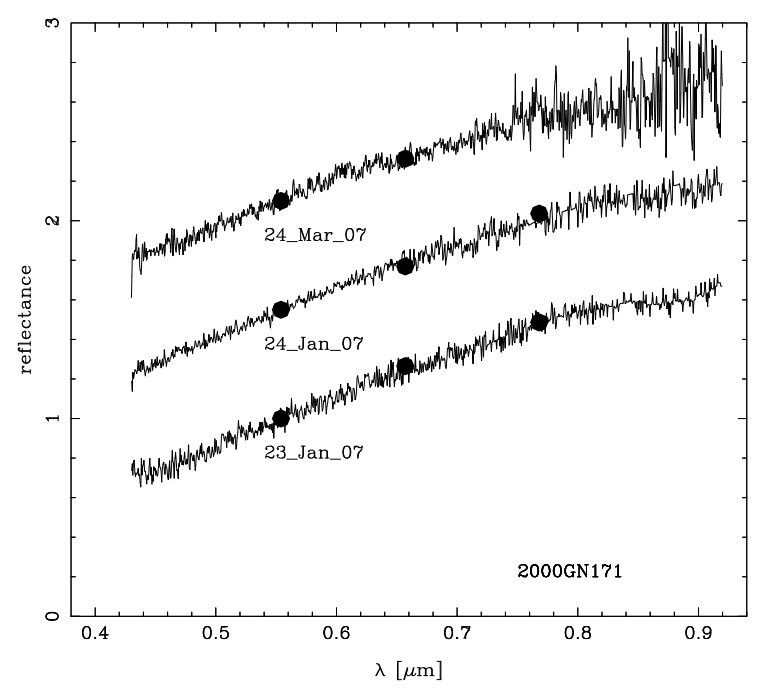

Fig. 4. Visible spectra of $47932\left(2000 \mathrm{GN}_{171}\right)$. Spectra are shifted vertically by 0.55 .

different figures showing the spectra, it is possible to see and compare the efficiency of both detector and their effect on our data. Especially useful is Fig. 5, which shows two Eris spectra taken in October 2006 and September 2007. They do not seem to show any spectral variation, but the fringing is quite evident above $0.7 \mu \mathrm{m}$ in the September 2007 observation. The details in the band at $0.9 \mu \mathrm{m}$ were totally lost in this last spectrum.

The fringing masks the features that could be present in the spectra, therefore making further modeling harder. With this in mind, our group has started using FORS2 to obtain visible data of the TNOs.

\section{Results}

We obtained a total of 25 spectra for 21 different objects including 5 Centaurs, 6 resonant objects, 8 classical TNOs, 2 detached objects, and 1 scattered disk object, following the classification scheme of Gladman et al. (2008, Table 1). The scheme follows an elimination algorithm. It defines a resonant object (Res) as any TNO captured in resonance with Neptune. If the object is neither resonant nor a comet and has a semi-major axis smaller

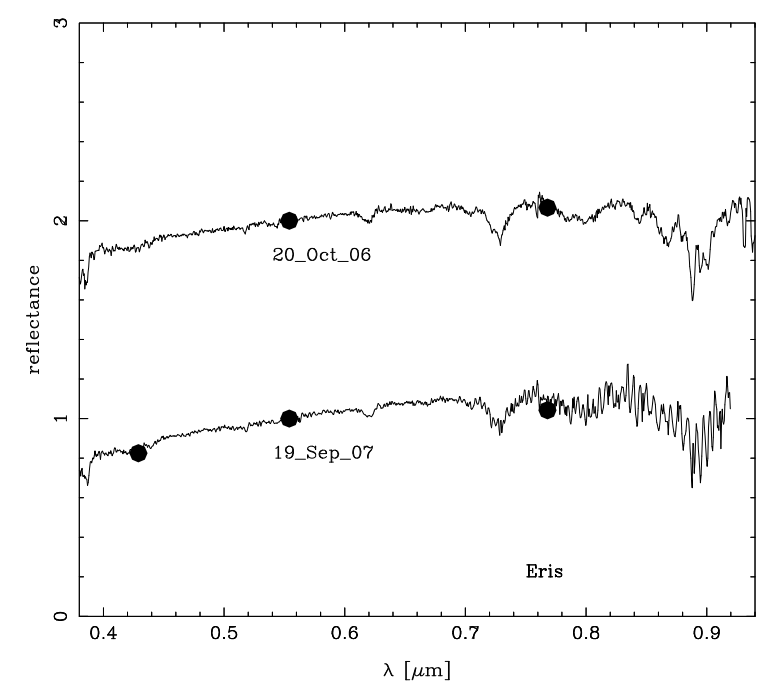

Fig. 5. Visible spectra of the dwarf planet 136199 Eris. Spectra are shifted vertically by 1.0 .

than that of Neptune, then it is a Centaur (Cen). If the TNO is none of the previous and is (has been) scattering (scattered) by Neptune, then it is a scattered disk object (SDO). If it is none of the previous, and if it has an eccentricity $>0.24$, then the object is a detached one (Det). Finally, if the object does not belong to any of the previous groups, the only remaining classification is classical object (Cla) (Gladman et al. 2008).

Most of the objects have almost featureless spectra. They can be characterized by an overall linear trend. Exceptions are 10199 Chariklo, 42355 Typhon, 136199 Eris, 2003 AZ $_{84}$ which will be discussed in the next section.

For each object we computed the slope $S$ of the spectral continuum using a standard least square technique for a linear fit in the wavelength range between 0.5 and $0.8 \mu \mathrm{m}$. The computed slopes and errors are listed in Table 1. The reported error bars take into account the $1 \sigma$ uncertainty of the linear fit plus $0.5 \%$ $(0.1 \mu \mathrm{m})^{-1}$ attributable to the use of different solar analog stars and detector efficiency.

From Table 1 we note that all objects have slopes ranging from neutral to very red. Our sample does not have any object with a negative slope. Figure 6 shows histograms of the spectral slopes. We grouped the objects into two subsets, one including Centaurs, scattered disk objects, and detached objects, and the other one the classical and resonant objects. These subsets are called, generically, samples 1 and 2 .

When considering the 21 objects, the median of the distribution is $20.0 \%(0.1 \mu \mathrm{m})^{-1}$. For objects with more than one observation we used a weighted mean. The distribution shows a lack of object with slope between 15 and $20 \%(0.1 \mu \mathrm{m})^{-1}$, but this may be due to a small number statistics. If we consider the two subsets independently we can see that most of the sample 1 objects have slopes $\leq 15 \%(0.1 \mu \mathrm{m})^{-1}$ except for a very red object, Crantor, which has a slope of about $40 \%(0.1 \mu \mathrm{m})^{-1}$. On the other hand, sample 2 has a larger number of red objects and few neutral ones.

Considering the dynamical classes mentioned in Table 1 we can see that four of the Centaurs have $S$ smaller than $12 \%$ $(0.1 \mu \mathrm{m})^{-1}$, while Crantor has the steepest slope of the sample. The resonant objects, considering all resonances together since the sample is too small to separate objects captured in different ones, have $S$-values higher than $20 \%(0.1 \mu \mathrm{m})^{-1}$. The classical objects have in general $S$-values higher than about $20 \%$ 

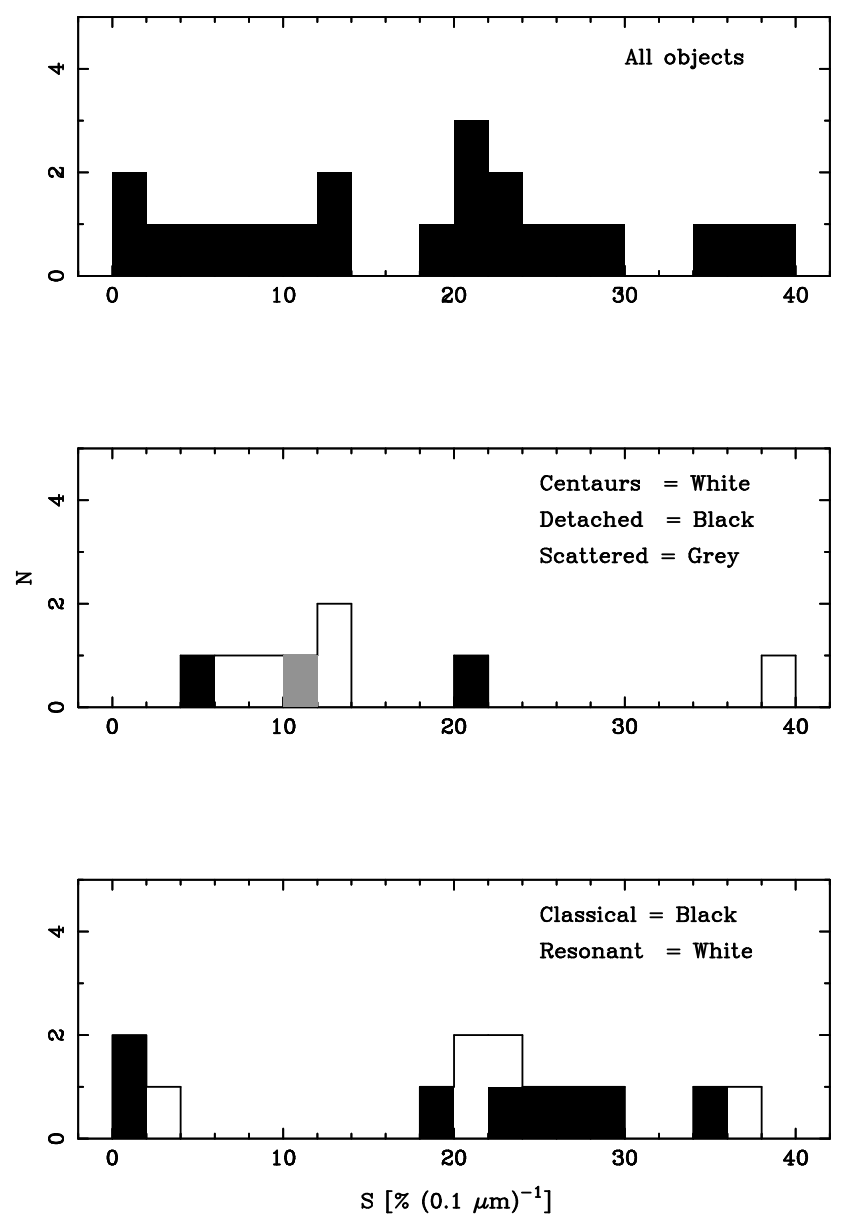

Fig. 6. $S$-Distributions for the complete sample (top), Centaurs plus detached plus scattered disk objects (middle), and classical plus resonant objects (bottom). The chosen bin was $2 \%(0.1 \mu \mathrm{m})^{-1}$.

$(0.1 \mu \mathrm{m})^{-1}$. The two objects that have small values of $S$ are both associated with the so-called family of $2003 \mathrm{EL}_{61}$ (Brown et al. 2007): 145453 (2005 $\mathrm{RR}_{43}$ ) and $2003 \mathrm{UZ}_{117}$, this last added to the list of probable family members by Ragozzine \& Brown (2007).

It is known that the classical objects show a relationship between the color (directly related to $S$ ) and the inclination of the orbit (see, for example, Doressoundiram et al. 2008). Objects with inclination lower than $5^{\circ}$ have a lack of blue-neutral slopes, while objects with inclination greater than $5^{\circ}$ display a large range of slopes. Our sample has only one low inclination object, $2002 \mathrm{KX}_{14}\left(I=0.4^{\circ}\right)$, which has a red slope. The rest of the objects show a large distribution of slopes, as expected.

Previous observations exist for some of these objects. The values of the measured slopes are presented in Table 2 . These values generally agree with ours, considering that different groups compute $S$ over slightly different spectral ranges and use different normalizations. The objects that present differences are $47932\left(2000 \mathrm{GN}_{171}\right)$ and 60558 Echeclus (2000 EC 98$)$.

\section{Discussion}

We discuss now some particularities that arose during the analysis of the data. We especially emphasize the features detected on some objects.
Table 2. Spectral slope values measured in this work compared to those already published.

\begin{tabular}{lcc}
\hline \hline Object & $\begin{array}{c}S\left(\%(0.1 \mu \mathrm{m})^{-1}\right) \\
\text { this work }\end{array}$ & $\begin{array}{c}S\left(\%(0.1 \mu \mathrm{m})^{-1}\right) \\
\text { from literature }\end{array}$ \\
\hline 10199 Chariklo & $7.8 \pm 0.5$ & $7.8 \pm 0.5^{a}$ \\
26375(1999 DE $)$ & $22.2 \pm 0.6$ & $25.5 \pm 0.2^{b}$ \\
28 978 Ixion & $20.0 \pm 0.8$ & $17.9 \pm 0.4^{c}$ \\
& & $22.2 \pm 0.2^{d}$ \\
32532 Thereus (2001 $\left.\mathrm{PT}_{13}\right)$ & $12.0 \pm 0.7$ & $11.1 \pm 0.2^{b}$ \\
47 171 (1999 $\left.\mathrm{TC}_{36}\right)$ & $37.3 \pm 0.7$ & $30.6 \pm 0.2^{b}$ \\
& & $29^{e}$ \\
47932(2002 $\left.\mathrm{GN}_{171}\right)$ & $21.8 \pm 0.4 \dagger$ & $24.1 \pm 0.2^{f}$ \\
& & $16.2 \pm 0.2^{g}$ \\
50 000 Quaoar & $28.5 \pm 0.8$ & $26.9 \pm 0.2^{f}$ \\
54 598 Bienor $\left(2000 \mathrm{QC}_{243}\right)$ & $8.7 \pm 0.7$ & $10.1 \pm 0.2^{b}$ \\
55 567 (2002 $\left.\mathrm{AW}_{197}\right)$ & $24.8 \pm 0.7$ & $22.1 \pm 0.2^{f}$ \\
60558 Echeclus $\left(2000 \mathrm{EC}_{98}\right)$ & $12.5 \pm 0.7$ & $23.7 \pm 0.7^{b}$ \\
83982 Crantor $\left(2002 \mathrm{GO}_{9}\right)$ & $39.1 \pm 0.8 \dagger$ & $35.8 \pm 0.1^{f}$ \\
145 453(2005 $\left.\mathrm{RR}_{43}\right)$ & $0.6 \pm 0.7$ & $0.4 \pm 1.0^{h}$ \\
2003 AZ & $3.0 \pm 0.6$ & $3.3 \pm 0.2^{f}$ \\
\hline
\end{tabular}

$\dagger$ Weighted mean from values in Table 1. ${ }^{a}$ Barucci et al. (1999), ${ }^{b}$ Lazzarin et al. (2003), ${ }^{c}$ Marchi et al. (2003), ${ }^{d}$ Boehnhardt et al. (2004), ${ }^{e}$ Merlin et al. (2005), ${ }^{f}$ Fornasier et al. (2004a), ${ }^{g}$ de Bergh et al. (2004), ${ }^{h}$ Pinilla-Alonso et al. (2007).

\section{1. $47932\left(2000 \mathrm{GN}_{171}\right)$}

The plutino $2000 \mathrm{GN}_{171}$ had revealed some intriguing absorption features in a spectrum taken in 2001 (Lazzarin et al. 2003), which were associated to materials that suffered aqueous alteration. In de Bergh et al. (2004) was presented a new spectrum taken in 2002, in which no features were detected. They proposed rotational heterogeneity as a possible explanation. To test this hypothesis, Fornasier et al. (2004a) attempted to take rotationally resolved spectra. They covered about $60 \%$ of the rotational phase without detecting significant features.

We present here three new spectra of $2000 \mathrm{GN}_{171}$, which do not show clear evidence of a feature at about $0.7 \mu \mathrm{m}$, thus we are not able to confirm its existence (Fig. 4). Our spectra are similar to those presented by Fornasier et al. (2004a), except for some absorption beyond $0.8 \mu \mathrm{m}$, which will be discussed later. They found a mean spectral slope of $(24.1 \pm 0.2) \%(0.1 \mu \mathrm{m})^{-1}$, which agrees with the values in Table 1.

The spectra taken in January 2007 cover about $25 \%$ of the rotational period $(P=8.329 \pm 0.005 \mathrm{~h}$, Sheppard \& Jewitt 2002). A simple computation, which should only be taken as indicative due to the uncertainty in the measured rotational period, shows that the spectrum taken in March 2007 corresponds to a different rotational phase (15\% apart from those of January). The total coverage $(\sim 30 \%)$ does not allow us to rule out possible rotational heterogeneities.

\subsection{Echeclus}

The value of $S$ for 60558 Echeclus is about $50 \%$ less than that of Lazzarin et al. (2003) which is, in principle, a difference too large to only be due to different conventions computing $S$. A simple visual comparison between our Fig. 2 and their Fig. 2 shows that the spectrum presented in this work is indeed not as red as theirs. Interestingly, our spectrum is similar to the photometric colors presented for Echeclus in Boehnhardt et al. (2002). They report an estimated spectral slope, derived from Echeclus's colors, of $(9.0 \pm 3.4) \%(0.1 \mu \mathrm{m})^{-1}$, which agrees with our slope 
Table 3. Central wavelengths of CH4-ice bands measured in the laboratory and in the spectrum of 136199 Eris, with uncertainties of about $0.0004 \mu \mathrm{m}$.

\begin{tabular}{ccc}
\hline \hline $\mathrm{CH}^{a}(\mu \mathrm{m})$ & This work $(\mu \mathrm{m})$ & Licandro et al. $(2006)(\mu \mathrm{m})$ \\
\hline 0.7296 & 0.7288 & 0.7296 \\
0.8897 & 0.8885 & 0.8881 \\
\hline
\end{tabular}

${ }^{a}$ Grundy et al. (2002).

to one sigma. One possible explanation for this discrepancy could be rotational heterogeneity, although more information is needed to confirm this hypothesis.

\subsection{Eris}

Eris was observed twice, first in October 2006 and then in September 2007. The spectra are presented in Fig. 5. Both of them are very similar, apart from the obvious problems of fringing in the September 2007's observation. The slopes $S$ are indistinguishable at one $\sigma$ level.

Absorption features due to methane ice are seen at 0.62 , $0.72,0.78,0.84,0.86$, and $0.89 \mu \mathrm{m}$. Comparing the central wavelengths of some bands with those of pure methane ice, as in Licandro et al. (2006), we detect a shift in the bands (see Table 3). A more detailed analysis of these spectra, as well as compositional models, will be presented by Merlin et al. (2008).

In addition, an absorption feature is detected around $0.39 \mu \mathrm{m}$, which cannot be due to methane ice (Fig. 5). The calibration star for both spectra on the figure is the same, the star Landolt SA93101 (or HD 11532), which is a G5V star. But when the Eris spectrum of September 2007 is divided by that of another G5V star, HD 2966, recorded during the same run (but not of as high a quality as that of SA93101), no feature is present. We conclude that the band at $0.39 \mu \mathrm{m}$ does not belong to Eris. It is probably due to slight differences in $\mathrm{CN}$ between our "solartype" star SA93101 and the Sun. Such differences have already been observed for stars that were otherwise considered good solar analogs (see, e.g., Hardorp 1978, 1980a,b).

\subsection{Family of $2003 E L_{61}$}

A group of TNOs having similar dynamical and observational characteristics was recently discovered. Two explanations were proposed: first, a carbon-depleted population (Pinilla-Alonso et al. 2007), whose surfaces cannot form an irradiation mantle, and second, a family of collisional origin, similar to the wellknown asteroidal families in the Main Belt (Brown et al. 2007).

We observed three objects that belong to this population. One of them is $2003 \mathrm{UZ}_{117}$, whose spectrum is flat without any evidence of features, similar to the one of 145453 (2005 RR 43 ), observed as well. The third object is 55565 (2002 AW 197$)$, which shows a red spectrum and therefore does not match the overall characteristics of this particular population.

\subsection{Observed features}

(i) We searched for the existence of subtle bands in the spectra in the region $0.5-0.8 \mu \mathrm{m}$, similar to those due to aqueous alteration. Following the method described in Vilas et al. (1993), we made a linear fit to the spectra and then removed this "continuum" from the spectra. We found three objects that present shallow and
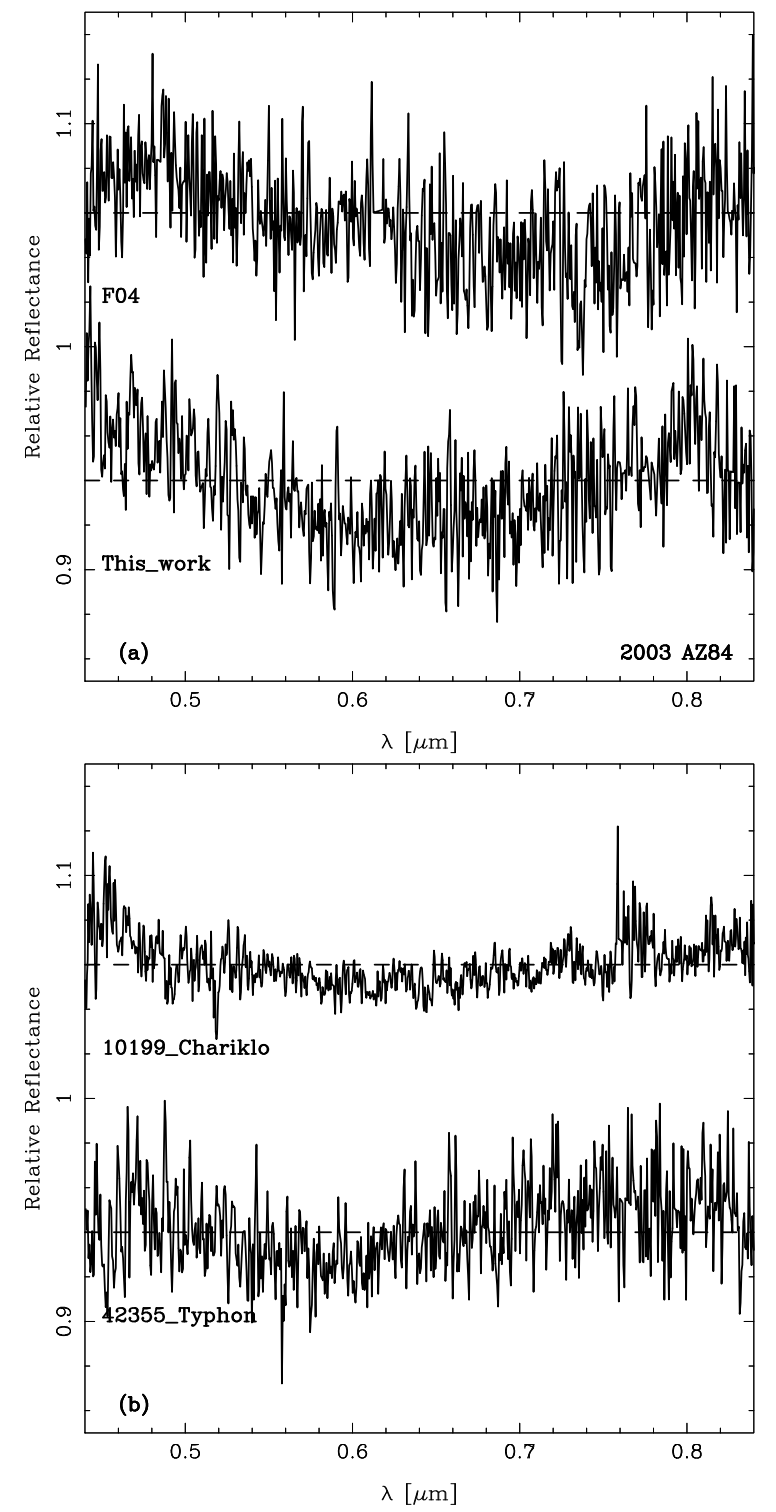

Fig. 7. a) Spectra of $2003 \mathrm{AZ}_{84}$ after removal of the continuum (see text). b) Spectra of 42355 Typhon and 10199 Chariklo after removal of the continuum.

broad features, in order of relevance: $2003 \mathrm{AZ}_{84}, 42355$ Typhon, and 10199 Chariklo.

On the first object, the same feature has already been reported by Fornasier et al. (2004a, Fig. 7, labeled F04). After performing a similar extraction of the continuum of the spectrum, a shallow broad band appeared similar to those due to aqueously altered materials. The spectrum presented in this paper resembles that of Fornasier et al. (2004a), but without the absorption band below $0.45 \mu \mathrm{m}$. We again detect a shallow broad band with a similar depth of about $2 \%$. The feature seems to be centered at about $0.65 \mu \mathrm{m}$ with a width of about $0.3 \mu \mathrm{m}$ (Fig. 7).

Typhon shows a feature that is different from that of the previous object. In Typhon's case the band is not so wide $(\sim 0.2 \mu \mathrm{m})$ and it is centered at shorter wavelengths $(\sim 0.6 \mu \mathrm{m})$. The depth with respect to the continuum is about $1.5 \%$. Finally, Chariklo's spectrum shows a similar shape to that of $2003 \mathrm{AZ}_{84}$, but with an even shallower feature (about 1\%). The spectrum of Chariklo in Fig. 7 shows a bending centered more or less at the same wavelength as $2003 \mathrm{AZ}_{84}$ and also a similar width. 
Could the analog stars be responsible for the features detected in these objects? There is no evident answer to this question, but we can try to discard possible systematic problems. The object 2003 AZ $_{84}$ was observed in two different epochs using different solar analogs, SA107998 in 2003 and SA1021081 in 2007 (both from Landolt's catalog). One possibility is that both stars are responsible for the presence of the band, but SA1021081 was also used to obtain the reflectance of 47932 (2000 $\left.\mathrm{GN}_{171}\right)$, which does not show the presence of a feature similar to that of $2003 \mathrm{AZ}_{84}$. A similar reasoning could be applied to the spectrum of 42355 Typhon. Unfortunately we do not have any spectrum obtained using SA107998. In the case of Chariklo, the analog star used was HD 107998, which was again used with $47932\left(2000 \mathrm{GN}_{171}\right)$ four days later. Once more there was no evidence of a similar absorption feature.

The mentioned values of the central wavelength and depth of the features depend on the extraction of the continuum, therefore they should be taken as indicative. Nevertheless, it is worth mentioning that we confirm the observation made by Fornasier et al. (2004a) for $2003 \mathrm{AZ}_{84}$ and that we detected weak features that need to be confirmed for two other objects.

The features discussed above resemble those found on the surface of dark asteroids of the Main Belt (see Vilas et al. 1994; Fornasier et al. 1999) and associated to aqueously altered materials. To have this kind of alteration, the presence of water is necessary in its liquid phase; or, in other words, there must be enough heat for the phase transition of the water. Some mechanisms have been proposed to explain this alteration in Main Belt asteroids, such as radiogenic heating or electrical induction during the T-Tauri phase of the Sun.

Unfortunately several questions arise when trying to explain aqueous alteration in TNOs. Little is known about the primordial content of, for instance, $\mathrm{Al}^{26}$, and electric induction is not efficient at the distances where the TNOs are thought to have been formed. A third option to explain the alteration are collisions in a denser, primitive trans-Neptunian region; nevertheless, it is not clear how collisions could provide enough heat. For a detailed discussion of the aqueous alteration of TNOs, see the comprehensive work of de Bergh et al. (2004).

(ii) A few objects have spectra that show a departure from a linear trend for wavelengths longer than $0.75 \mu \mathrm{m}$. These objects are 26375 (1999 $\left.\mathrm{DE}_{9}\right), 47932$ (2000 GN 171$), 55565$ (2002 AW 197 ), 55637 (2002 $\mathrm{UX}_{25}$ ), 120132 (2003 FY 128 ), and 145452 (2005 $\mathrm{RN}_{43}$ ). They were observed in different epochs, using different analog stars and with different setups of the instrument (see Sect. 2). In particular, note the case of 47932 ( $2000 \mathrm{GN}_{171}$ ) that shows almost the same spectral behavior on three different nights and using two different solar analogs.

The drop in the reflectance starts at about $0.75 \mu \mathrm{m}$, even for those objects that show the fringing pattern. To measure it we followed the method described above, but with slight differences. As most of the spectra show a relatively linear behavior between 0.5 and $0.7 \mu \mathrm{m}$ we estimate the continuum by a linear fit between this range and remove it from the spectra by a division. On the resulting normalized spectra, we compute the quantity $D_{0.8}$ which is the difference between 1 and the normalized reflectance at $0.8 \mu \mathrm{m}$, expressed in percentage. The values appear in Table 1.

It is important to say that $D_{0.8}$ is a rather arbitrary value, which we defined in order to quantify the apparent departure from the linearity of the observed spectra. Had we defined $D$ at 0.85 or $0.9 \mu \mathrm{m}$, it would have changed the results quantitatively but not qualitatively. A good reason to choose the wavelength $0.8 \mu \mathrm{m}$ is that it corresponds to a region where all the spectra have a relatively good signal-to-noise ratio; longer wavelengths would be less useful due to the significant fringing in some of the spectra. The first interesting thing to mention is that all the three objects (and only those) discussed above, Chariklo, Typhon, and $2003 \mathrm{AZ}_{84}$ have negative values of $D_{0.8}$, which is consistent with an overall concave shape.

We explored the possibility that the change in slope is related to a general flattening of the spectra beyond $0.8 \mu \mathrm{m}$. We compared the values of $D_{0.8}$ with the available $V-J$ colors (Fulchignoni et al. 2008), but we did not find any clear relationship. Moreover, there is no direct relationship between $D_{0.8}$ and the airmass, seeing, or spectral slope. To conclude, we did not find any definitive explanation for the effect. One possibility is the combination of large seeing and incomplete correction of atmospheric extinction. Either way, a compositional explanation cannot be discarded.

(iii) Some objects show subtle features at wavelengths longer than $0.80 \mu \mathrm{m}$, among them: $47932\left(2000 \mathrm{GN}_{171}\right)$ (2007 January 23 spectrum) and 120132 (2003 FY 128 ). Both spectra show absorption features at about $0.865 \mu \mathrm{m}$. In this region bands could appear that are caused by hydrated minerals (Vilas et al. 1994) but that are very faint. Due to the decrease in the signal-to-noise ratio and an incomplete removal of the strong telluric bands after $0.8 \mu \mathrm{m}$ in this region of the spectra, together with possible undesirable effects caused by the sorting filter, a compositional interpretation should wait for further observations.

\section{Conclusions}

As part of a large program of observations of trans-Neptunian objects, we have presented new visible spectra of 21 objects, including 7 objects without previous published data. The main conclusions are:

- Classical TNOs in our sample are redder than the Centaurs. These two populations tend to cluster around different values, with notable exceptions: the Centaur 83982 Crantor, which is the reddest object in our sample, and the classical TNOs 145453 (2005 RR 43 ) and $2003 \mathrm{UZ}_{117}$, which are neutral. The last two objects dynamically belong to the so-called family of $2003 \mathrm{EL}_{61}$.

- A few objects show, or have evidence of, features in their visible spectra. The most prominent case is 136199 Eris, whose spectral bands are displaced with respect to pure methane-ice, confirming previous works. A few objects seem to have shallow and broad spectral bands similar to those present in low-albedo asteroids due to the presence of wateraltered materials. We confirm the detection seen by Fornasier et al. (2004a) on $2003 \mathrm{AZ}_{84}$ and present new detections on 10199 Chariklo and 42355 Typhon, which remain to be confirmed.

Some questions remain open. First, what is the reason for the departure from linearity for some spectra? Second, are the detected features real? Last, but not least, it remains to further study the possible rotational heterogeneity of $47932\left(2000 \mathrm{GN}_{171}\right)$ and 60558 Echeclus.

The result presented in this work are the first part of the new large program. Ongoing observations will increase the sample and confirm or disprove the main results. It is out of the scope of this paper to perform a detailed discussion linking the observational results to origin/collisional models. A detailed statistical study of the complete set of observations of the program, as 
well as of previously published data, and the possible relationship with orbital and physical parameters will be presented at the end of this program.

Acknowledgements. A.A.C. would like to thank the ESA program of international traineeship for financial support. We thank F. DeMeo for kindly reviewing the English of the manuscript. We would like to thank the anonymous referee, whose suggestions and comments much improved this paper.

\section{References}

Barucci, M. A., Lazzarin, M., \& Tozzi, G. P. 1999, AJ, 117, 1929

Boehnhardt, H., Delsanti, A., Barucci, M. A., et al. 2002, A\&A, 395, 297

Boehnhardt, H., Bagnulo, S., Muinonen, K., et al. 2004, A\&A, 415, L21

Brown, M. E. 2008, The Largest Kuiper Belt Objects, in The Solar System beyond Neptune, ed. A. Barucci, H. Boehnhardt, D. Cruikshank, \& A. Morbidelli (Tucson: Univ. of Arizona Press), 335

Brown, M. E., Barkume, K. M., Ragozzine, D., et al. 2007, Nature, 446, 294

de Bergh, C., Boehnhardt, H., Barucci, M. A., et al. 2004, A\&A, 416, 791

DeMeo, F., Fornasier, S., Barucci, M. A., et al. 2008, in preparation

Doressoundiram, A., Boehnhardt, H., Tegler, S. C., et al. 2008, Color Properties and Trends of the Transneptunian Belt, in The Solar System beyond Neptune, ed. A. Barucci, H. Boehnhardt, D. Cruikshank, \& A. Morbidelli (Tucson: Univ. of Arizona Press), 91

Fornasier, S., Lazzarin, M., Barbieri, C., et al. 1999, A\&AS, 135, 65
Fornasier, S., Doressoundiram, A., Tozzi, G. P., et al. 2004a, A\&A, 421, 353 Fornasier, S., Dotto, E., Marzari, F., et al. 2004b, Icarus, 172, 221

Fulchignoni, M., Belskaya, I., Barucci, M. A., et al. 2008, Transneptunian Object Taxonomy, in The Solar System beyond Neptune, ed. A. Barucci, H. Boehnhardt, D. Cruikshank, \& A. Morbidelli (Tucson: Univ. of Arizona Press), 181

Gladman, B., Marsden, B. G., \& VanLaerhoven, C. 2008, Nomenclature in the outer Solar System, in The Solar System beyond Neptune, ed. A. Barucci, H. Boehnhardt, D. Cruikshank, \& A. Morbidelli (Tucson: Univ. of Arizona Press), 43

Grundy, W., Schmitt, B., \& Quirico, W. 2002, Icarus, 155, 486

Hardorp, J. 1978, A\&A, 63, 383

Hardorp, J. 1980a, A\&A, 88, 334

Hardorp, J. 1980b, A\&A, 91, 221

Landolt, A. U. 1992, AJ, 104, 340

Lazzarin, M., Barucci, M. A., Boehnhardt, H., et al. 2003, AJ, 125, 1554

Licandro, J., Grundy, W. M., Pinilla-Alonso, N., et al. 2006, A\&A, 458, L5

Marchi, S., Lazzarin, M., Magrin, S., et al. 2003, A\&A, 408, L17

Merlin, F., Barucci, M. A., Dotto, E., et al. 2005, A\&A, 444, 977

Merlin, F., Alvarez-Candal, A., Delsanti, A., et al. 2008, AJ, submitted

Pinilla-Alonso, N., Licandro, J., Gil-Hutton, R., et al. 2007, A\&A, 468, L25

Ragozzine, D., \& Brown, M. E. 2007, AJ, 134, 2160

Sheppard, S. S., \& Jewitt, D. C. 2002, AJ, 124, 1757

Tegler, S. C., Grundy, W. M., Romanishin, W., et al. 2007, AJ, 133, 526

Vilas, F., Hatch, E. C., Larson, S. M., et al. 1993, Icarus, 102, 225

Vilas, F., Jarvis, K. S., \& Gaffey, M. J. 1994, Icarus, 109, 274 